\title{
PERFILES DE CONSUMO TELEVISIVO: UN ESTUDIO TRANSCULTURAL
}

\author{
(TELEVISION VIEWING PROFILES: A TRANSCULTURAL STUDY)
}

Concepción Medrano

Juan Ignacio Martínez de Morentín

Pedro Apodaca

Universidad del País Vasco (UPV/EHU)

DOI: $10.5944 / e d u c X X 1.14606$

\section{Cómo referenciar este artículo/How to reference this article:}

Medrano, C.; Martínez de Morentín, J. I. y Apodaca, P. (2015). Perfiles de consumo televisivo: Un estudio transcultural. Educación XX, 18(2), 305-321, doi: 10.5944/educXX1.14606

Medrano, C.; Martínez de Morentín, J. I., \& Apodaca, P. (2015). Perfiles de consumo televisivo: Un estudio transcultural [Television viewing profiles: A transcultural study]. Educación $X X 1,18(2)$, 305-321, doi: 10.5944/educXX1.14606

\section{RESUMEN}

En esta investigación se parte de la idea básica de que el medio televisivo es un agente de socialización en la adolescencia. Su objetivo general fue conocer algunos indicadores del perfil de consumo televisivo en una muestra transcultural de adolescentes. La muestra total estuvo compuesta por 1.238 participantes, pertenecientes a ocho regiones de diversos países. El instrumento de medida utilizado fue el cuestionario de hábitos televisivos (CH-TV.02). La recogida de datos se realizó a través de una plataforma on-line y presencial. Respecto a los hallazgos encontrados, existen diferencias transculturales y significativas en los indicadores estudiados: actividades alternativas, razones de elección del personaje, identificación con el personaje favorito y realismo percibido. En el análisis de resultados, destaca como actividad alternativa a ver la televisión estar con la familia, siendo la lectura una de las actividades a las que menos horas dedican. Así mismo eligen a su personaje favorito, sobre todo, por su simpatía y humor y se identifican con él porque intentan ver las cosas desde su punto de vista. Perciben que la televisión es muy realista en cómo presenta las consecuencias de las drogas y el alcohol y muy poco realista en cómo presenta las situaciones escolares. Las narraciones mediáticas, desde el punto de vista educativo, deberían trabajarse en las aulas con el fin de evitar una recepción pasiva y favorecer la decodificación de los mensajes. 


\section{PALABRAS CLAVE}

Adolescentes; estudio transcultural; televisión; actividades alternativas; razones de elección; identificación; realismo percibido.

\section{ABSTRACT}

The basic hypothesis of this research project is that television is a force for socialization during adolescence. The general aim was to explore some of the indicators of the television viewing profile in a cross-cultural sample of adolescents. The total sample group comprised 1,238 subjects from eight regions in different countries. The measurement instrument used was the television viewing habits questionnaire (CH-TV.02). The data was collected both by means of an on-line platform and in person. The results revealed significant cross-cultural differences in all the indicators studied: alternative activities, reasons for choosing their favorite character, identification with their favorite character and perceived realism. Of special interest were the results regarding alternative activities to watching television, in which reading was one of the activities that subjects said they spent least time engaged in. Their favorite characters were mostly chosen for their friendliness and humor, and they identify with them because they try to see things from their point of view. They see television as being very realistic in how it presents the consequences of drugs and alcohol and very unrealistic in how it presents school situations. From an educational perspective, media narratives should be studied in the classroom in order to avoid mere passive reception and foster adolescents' ability to decode the messages conveyed.

\section{KEY WORDS}

Adolescents; cross-cultural study; television; alternative activities; reasons for choosing; identification; perceived realism.

\section{INTRODUCCIÓN}

En la actualidad los medios de comunicación y, en concreto, la televisión es considerada en la literatura científica como un agente, entre otros, de socialización en nuestra juventud. Ahora bien, a pesar de que la socialización se desarrolla fundamentalmente a través de la familia y el grupo de iguales, como ya han demostrado diversos autores, la irrupción de los medios ha significado un cambio importante en el equilibrio aportado por los agentes clásicos y en la construcción de valores (Cortina, 2004; Medrano, Cortés y Palacios, 2007; Negroponte, 1996). Incluso algunos autores como Sartori (1998) han afirmado, quizá de forma algo extrema, que la televisión 
genera un nuevo ser humano y que es la más grande agencia de formación de la opinión pública. No obstante, más allá de las teorías del análisis de los efectos y de la aculturación, ambas de corte positivista, así como los iniciales planteamientos críticos de la Escuela de Francfort, los estudios culturales iniciados en el Centro de Birmingham, dotan de una mayor relevancia a los procesos socio-culturales que tienen lugar en la recepción de los mensajes (Mattelart y Mattelart, 1997; Medrano, 2008).

De acuerdo con Martín (1997), y desde la teoría de la recepción, los medios de comunicación participan en la construcción de la identidad de nuestros adolescentes y ellos, también, son agentes activos en la elección de los contenidos que aparecen en las distintas pantallas, tanto por el tiempo dedicado a la mismas como por la influencia socializadora a través de la identificación con sus personajes preferidos (Orozco, Navarro y García, 2012; Pindado, 2006).

Ahora bien, dado que la adolescencia es un período crítico en el desarrollo personal que se caracteriza por un conflicto entre la seguridad proporcionada por la familia y la incertidumbre de salir al mundo, los medios y, en concreto, la televisión ponen a disposición de los adolescentes un conjunto de recursos simbólicos que les permitirán desarrollar aspectos tan importantes como la identidad de género, el aprendizaje social y, la construcción de valores con la ayuda de la educación familiar y la mediación televisiva. (Cánovas y Sahuquillo, 2010; Elzo, 2006).

Así puede ser el caso del consumismo que, de acuerdo con Castillejo, Colom, Alonso, Rodríguez, Sarramona, Touriñán y Vázquez, (2011) serían los propios medios de comunicación los que se convierten en moduladores de ciertas conductas dado que la seducción publicitaria se genera a través de un proceso de copia de identidades mediáticas, sin olvidar que la propia publicidad explota las motivaciones y tendencias ya instaladas en los jóvenes. En este sentido, la propuesta de Colom (1998) de una educación para el desarrollo encajaría de forma adecuada para trabajar los contenidos narrativos de la televisión desde su vertiente formativa.

No obstante, es preciso señalar que muchos de los estudios sobre audiencia se han realizado con un objetivo comercial y de acuerdo con Vera (2001) los efectos configuradores de los medios en el contexto neoliberal nos exigen reconocer su gran influencia y educar en los medios de comunicación. Lo relevante en la investigación educativa es situar dicho consumo en un marco pedagógico y conocer los perfiles televisivos con el fin de poder afirmar si la televisión es tan perniciosa como se afirma y, en nuestro caso, conocer si existen diferencias transculturales. 
En concreto, en este trabajo se ha tratado de conocer el perfil de consumo televisivo, a través de algunos indicadores en una muestra transcultural. Cabe señalar que en la revisión de los trabajos previos sobre el impacto de la televisión en la construcción de la identidad, se observan, en ocasiones, conclusiones ambivalentes y con ciertas lagunas. Lo que sí aparece de forma nítida es que no es tanta la televisión que se visualiza; aunque, en los contenidos televisivos y en la transmisión de sus mensajes, se percibe una ambigüedad que es lesiva desde el punto de vista del desarrollo moral de los adolescentes (Medrano, Aierbe y Martínez de Morentin, 2011; Rodríguez, Medrano, Aierbe y Martínez de Morentin, 2013).

Pérez Alonso-Geta (2003) en el análisis acerca de la influencia de los contenidos televisivos en la juventud, halló que los niños de la Comunidad Valenciana ven los mismos programas que los adultos. Esta autora concluye que a través de la pantalla los niños aprenden a comportarse y, por tanto, el medio es un importante agente de socialización.

Otro aspecto, muy relacionado con el anterior, son las horas que dedican los chicos y chicas a otras actividades diferentes a ver la televisión. En los estudios previos, en general, los adolescentes practican con mayor asiduidad aquellas actividades que suponen un escaso sacrificio personal. Así, por ejemplo, en un estudio del Ararteko (2009), las medias más altas se encuentran en: escuchar música, salir con amigas/os, navegar por Internet, estar con los padres y hacer uso de videojuegos. Mientras que actividades como la lectura (tanto de cómics como de libros), que pudieran suponer un relativo esfuerzo mental, pasan a un segundo plano.

Según el Instituto Nacional de la Juventud, estamos ante una juventud algo más pasiva que en años anteriores. Sirva como ejemplo el hecho de que el ejercicio del deporte ocupaba un lugar entre las tres primeras actividades que decían realizar en 2001, cuando en los últimos años aparece como décima o undécima actividad elegida o deseada (INJUVE, 2008).

Los consumos de televisión ofrecen, asimismo, perfiles bajos: jóvenes inactivos, con menor formación y más dependientes. Según el Instituto Nacional de Consumo, la dedicación al trabajo, como promedio, no alcanza las siete horas y la dedicación al estudio representa tres horas y media, mientras que a estar con la familia se le dedica menos tiempo que a estar con los amigos o con el novio o la novia. La dedicación a la televisión se aproxima a las dos horas y la lectura está en torno a una hora y media. (Martín-Serrano, 1998).

Lo que también aparece de forma nítida en los estudios previos es que la televisión se perfila como un agente de socialización en la formación de 
la identidad de chicos y chicas. La investigación demuestra, que la identificación con los personajes es un factor a tener en cuenta en la explicación de la construcción de la identidad (Cohen, 2006).

La presente investigación se ha realizado con una muestra transcultural de adolescentes y la principal crisis que debe afrontar durante esta etapa es la crisis de la identidad. Sin embargo, los chicos y chicas en este periodo vital no se forman una identidad "copiando" a otros modelos, sino que es necesario modificar y sintetizar identidades. En este sentido, la televisión, entre otros muchos agentes, puede ser a través de la identificación con sus personajes y el realismo percibido en dicho medio, una fuente más que contribuye a la construcción de su identidad. Se dispone de datos empíricos que nos indican cómo los adolescentes utilizan los medios de comunicación para comprender el mundo y para buscar modelos con los que identificarse (Sahuquillo, 2007). Así mismo, existe evidencia empírica respecto a cómo durante la adolescencia se tiende a que los personajes admirados sean modelos que aporten sentido a la formación de la identidad en esta época del desarrollo vital (Guarinos, Gordillo, Ramírez, Jiménez-Varea y Hermida, 2010).

En concreto, los datos expuestos por Hoffner (1996), confirman que los adolescentes tienden a identificarse con personajes del mismo género y edad, siendo uno de los rasgos que señalan la personalidad e inteligencia. Medrano, Cortés, Aierbe y Orejudo (2010), en un estudio sobre la elección de sus personajes preferidos, en una muestra con adolescentes, encuentran que estos se inclinan, sobre todo, por personajes divertidos. En esta misma línea Pérez y Torres (2012) hallan que las características personales referidas a la personalidad e inteligencia, al igual que las referidas a los rasgos físicos del personaje son las más señaladas por los adolescentes.

Por otra parte, la investigación realizada por Calvert, Murray y Conger (2004), con chicos y chicas taiwaneses y estadounidenses, cuyo objetivo principal era conocer los personajes con los que se identifican los jóvenes así como las características principales que los caracterizan, hallan que los jóvenes taiwaneses y estadounidenses, encuentran más atractivas las características prosociales de sus personajes favoritos, así como la personalidad.

Así mismo, otro aspecto a considerar es el grado de realismo percibido en la representación de temas de actualidad; dado que, también, puede contribuir a la construcción de la identidad del adolescente. Butcher (2010) analizó la credibilidad de las representaciones culturales de los latinos en los programas de televisión en inglés y demostró que los mexicanos de segunda y tercera generación que viven en California perciben que la televisión ame- 
ricana presenta los extremos culturales de forma exagerada y que no reflejan la forma en que experimentan su identidad latina.

Cabe destacar que cuando los telespectadores se implican emocionalmente con los contenidos televisivos, tienden a identificar un documental ficticio como realista; mientras que, los participantes que no están implicados emocionalmente tienden a argumentar que es el documental basado en hechos reales el que presenta situaciones más realistas (Quick 2009).

Por tanto, se puede concluir que: a) cuanto mayor es el realismo percibido mayor es el efecto incubado; b) las series, les ofrecen la oportunidad de hablar sobre sí mismos y clarificar ideas sobre la identidad de género y sexual; c) la identificación con los personajes famosos les ofrece un grupo secundario de seudo amigos; d) la identificación con personajes famosos favorece el desarrollo de su autonomía personal.

A partir del estado de la cuestión, en este trabajo se han planteado los siguientes objetivos específicos:

1. Indagar respecto a las diferencias encontradas en actividades alternativas a ver la televisión en los ocho contextos estudiados.

2. Describir las razones de elección de su personaje favorito en los adolescentes en relación al origen cultural.

3. Conocer el grado de identificación con su personaje favorito en los adolescentes en relación al origen cultural.

4. Indagar acerca del grado de realismo que perciben en el medio televisivo así como la representación de diferentes temas de actualidad social y las diferencias en relación al origen cultural.

\section{MUESTRA}

La muestra total asciende a 1238 sujetos distribuidos en 8 regiones diferentes; tres en el contexto español (Aragón, N=183; Andalucía, N=125; País Vasco, $\mathrm{N}=184$ ), cuatro en el contexto latinoamericano (Cibao, —República Dominicana- $\mathrm{N}=148$; Jalisco, -México- $\mathrm{N}=150$; O’Higggins, -Chile$\mathrm{N}=145$; Oruro,-Bolivia-, $\mathrm{N}=197$ ) y una en el contexto irlandés (Dublín, $\mathrm{N}=106)$. En cuanto al género, 545 son hombres y 676 mujeres.

El muestreo utilizado ha sido de "conveniencia» pues el estudio prioriza la validez ecológica (validez externa de situación) a la estricta represen- 
tatividad aleatoria de la muestra utilizada (validez externa de población). De esta manera, se han seleccionado centros que ofrecieran las mejores condiciones de accesibilidad y de aplicación de las pruebas. Sin embargo, se ha procurado la equivalencia entre los grupos estudiados en las diferentes regiones tomando criterios similares de selección de centros y de grupos de dichos centros: tipo de centro, edad y curso.

Esta orientación en la selección de la muestra es la adecuada en el marco de los propósitos de este estudio. No pretende estimar tasas en la población sino realizar comparaciones entre distintos grupos culturales.

La muestra recogida ha sido sometida a un proceso sistemático de filtrado y depuración con el fin de garantizar la calidad de la información recogida. Se han utilizado dos criterios para la depuración de casos: a) sujetos que dejan de contestar más del $50 \%$ de las preguntas y b) sujetos con respuestas contradictorias en un número elevado de preguntas.

El alumnado seleccionado estudia en $4 .^{\circ}$ de la ESO y $2 .^{\circ}$ de bachillerato o lo que corresponde al contexto latinoamericano a PREPA y/o primer y tercer grado de bachillerato. Respecto al tipo de centro, la aplicación se ha realizado en dos o más centros para cada submuestra, bien sean centros públicos y/o privados o centros con diferentes niveles socioeconómicos, que no sean extremos. Debido a las limitaciones presupuestarias de este tipo de proyectos la representatividad de la muestra no podía apoyarse en sistemas de selección aleatoria y las regiones de los ocho países fueron seleccionadas por la participación en el proyecto de investigadores de dichos países.

\section{MÉTODO}

Esta investigación es un estudio ex post-facto, descriptivo-correlacional y transcultural. Estudia algunos indicadores del perfil de consumo en una muestra transcultural de adolescentes con edades comprendidas entre $\operatorname{los} 14$ y 19 años.

\section{Instrumentos de medida}

El instrumento, utilizado para recoger el perfil y/o consumo televisivo, es el Cuestionario de Hábitos Televisivos (CH-TV.02), creado por el equipo de investigación de la Universidad del País Vasco y que ha sido modificado y simplificado tras la prueba piloto. El cuestionario final consta de siete preguntas iniciales que recogen datos referidos a: estudios, profesión y situación actual del padre y de la madre; así como datos que nos dan información 
sobre la estructura familiar: personas con las que se convive, género y edad de los hermanos y el lugar que se ocupa entre los mismos. Posteriormente se presentan 24 ítems que recogen un total de 14 indicadores.

Para este trabajo se han estudiado los siguientes indicadores: actividades alternativas (objetivo 1); razones para la elección de su personaje favorito (objetivo 2) grado de identificación con su personaje favorito (objetivo 3) y realismo televisivo (objetivo 4).

Actividades alternativas. Se refiere a las horas que dedican a ocho alternativas diferentes a ver la TV en su tiempo libre (ítem 7).

Razones para la elección de su personaje favorito e identificación. Razones por las que mejor le cae su personaje favorito, (ítem 21), así como el grado de identificación (ítem 12).

Realismo televisivo. Se refiere al grado de realismo que los adolescentes perciben en la TV, respecto a la representación de diferentes temas de actualidad: (ítem, 23).

\section{Procedimiento}

Para la recogida de los datos, en primer lugar, se adaptó el cuestionario de la versión española para generar las nuevas versiones en los otros cuatro contextos estudiados. En la adecuación de las versiones (mexicana, boliviana, chilena e inglesa) se tuvo en cuenta en todo momento que no cambiara el sentido de las preguntas realizadas Para ello, el cuestionario CH-TV.02 fue revisado por ocho expertos antes de su elaboración definitiva, quienes, entre otros aspectos, valoraron si las preguntas relacionadas con los hábitos televisivos eran aplicables y tenían sentido a cada cultura.

En segundo lugar y una vez realizada y comprobada la validez de cada adaptación, se procedió a la toma de contacto con el profesorado de los centros previamente seleccionados. La selección de los centros como se ha citado anteriormente, fue por conveniencia y teniendo en cuenta que no pertenecieran a niveles socioeconómicos extremos. Se explicó a cada profesor/a ayudante, cual debía de ser el procedimiento a seguir para aplicar el cuestionario, así como la edad del alumnado (14-19 años). De este modo, el cuestionario se realizó on-line en todos los centros y presencialmente. El profesor/a ayudante realizaba la prueba con sus alumnos/as en el aula de ordenadores del propio centro escolar. La aplicación del cuestionario conlleva un total de 50-60 minutos 
Se obtuvo, también, el consentimiento informado de los padres, madres y alumnado.

\section{ANÁLISIS DE DATOS}

En lo que respecta al primer objetivo, horas semanales que dedican a otras actividades diferentes a ver la televisión, se observa, en la tabla 1, que respecto a los valores medios, las puntuaciones más altas las encontramos en estar con la familia $(M=9,42)$, seguida de estar con los amigos $(M=8,96)$ y en tercer lugar y cuarto lugar, escuchar música $(\mathrm{M}=7,35)$ y navegar por Internet $(M=6,90)$. Mientras que a los videojuegos es la actividad a la que menos horas dedican $(M=2,48)$ seguida de leer $(M=2,72)$. Desde un análisis más cualitativo y relacionando estos datos con los expuestos en el primer objetivo, ver la televisión ocuparía uno de los últimos lugares en cuanto a tiempo que dedican los adolescentes a distintas actividades en su tiempo libre.

Tabla 1

Estadísticos descriptivos: alternativas a ver la televisión en la muestra total

\begin{tabular}{lccc}
\hline $\begin{array}{c}\text { Alternativas a ver la televisión: } \\
\text { horas semanales }\end{array}$ & & Media & Desviación típica \\
\hline Leer & 1205 & 2,72 & 3,042 \\
Navegar por Internet & 1217 & 6,90 & 5,340 \\
Utilizar videojuegos & 1190 & 2,48 & 3,765 \\
Hacer deporte & 1202 & 4,71 & 4,433 \\
Escuchar música & 1219 & 7,35 & 5,665 \\
Estar con la familia & 1205 & 9,42 & 6,167 \\
Estar con los amigos & 1213 & 8,96 & 5,678 \\
Usar el móvil & 1214 & 6,53 & 6,533 \\
Otros - Horas de dedicación & 935 & 5,33 & 5,468 \\
Región & 1238 & 4,46 & 2,393 \\
\hline
\end{tabular}

Con el objeto de analizar la significatividad respecto a las diferencias contextuales se realizó la prueba de Kursal-Walis. Los resultados muestran un efecto significativo en el valor estadístico Chi-cuadrado $\chi^{2}{ }_{(7)}$ en todas las actividades. Hay que destacar que estar con la familia, es la actividad a la que más horas dedican. Si nos referimos a la segunda actividad a la que más tiempo dedican, estar con los amigos, las diferencias, también, son significativas con un $\left(\chi_{(7)}^{2}=105,427, \mathrm{p}=.000\right)$. En este caso, es el País Vasco el que presenta el rango más alto 751,84, mientras que en $\mathrm{O}^{\prime}$ Higgins se halla un rango de 469,38. Así mismo son destacables las diferencias encontradas res- 
pecto a la utilización del móvil $\left(\chi_{(7)}^{2}=244,915, \mathrm{p}=.000\right)$; Dublín, por ejemplo, presenta un rango medio en dicha prueba de 974,04 mientras que el País Vasco presenta un rango medio de 431,75. Si nos referimos a las horas que dedican a la lectura $\left(\chi^{2}{ }_{(7)}=88,408, \mathrm{p}=.000\right)$, el rango más alto se halla en $\mathrm{O}^{\prime}$ Higgins 619,26 y el más bajo en Jalisco 466,70.

En el segundo objetivo, tal y como se puede observar en la Tabla 2 la razón más esgrimida de elección de su personaje favorito, considerada la muestra total, es por su simpatía y humor $(M=4,94)$ seguida de por su personalidad $(M=4,84)$ y en último lugar por su actitud no conformista y rebelde $(\mathrm{M}=3,50)$.

Tabla 2

Medias transculturales respecto a las razones de elección de su personaje favorito

\begin{tabular}{lcccccccccc}
\hline \multicolumn{10}{c}{ REGIÓN } \\
\hline \multicolumn{1}{c}{$\begin{array}{c}\text { Razones } \\
\text { de elección }\end{array}$} & $\begin{array}{c}\text { País } \\
\text { Vasco }\end{array}$ & Aragón & Andalucía & Dublín & Jalisco & Cibao & Oruro O’Higgins & Total \\
\hline Por el atractivo físico & 2,94 & 3,51 & 3,34 & 3,75 & 3,89 & 4,02 & 3,46 & 3,55 & 3,53 \\
Por su inteligencia & 3,69 & 4,08 & 4,11 & 3,75 & 4,10 & 4,84 & 4,31 & 3,94 & 4,11 \\
Por su simpatía y humor & 4,68 & 5,07 & 5,04 & 4,57 & 4,99 & 5,06 & 4,93 & 5,13 & 4,94 \\
Por su personalidad & 4,42 & 4,87 & 4,85 & 4,62 & 5,02 & 4,93 & 4,93 & 5,11 & 4,84 \\
Por su trabajo & 3,41 & 3,80 & 4,06 & 3,71 & 4,18 & 4,52 & 4,29 & 4,14 & 4,00 \\
Por su actitud no & 3,23 & 3,70 & 3,92 & 3,80 & 3,70 & 2,79 & 3,64 & 3,29 & 3,50 \\
conformista y rebelde & 3,23 & & & & & & & & &
\end{tabular}

Al tratar de comprobar, mediante la prueba Anova, si dichas diferencias son significativas se halla que todas las diferencias transculturales son estadísticamente significativas (p'.001), aunque ciertamente son moderadas. Hay que exceptuar el argumento de por su trabajo y por su inteligencia ambas con un $\left(\eta^{2}=.045\right)$. Respecto a ser la inteligencia la razón por las que le cae mejor un personaje, la puntuación más alta se obtiene en Cibao (4.84) y la más baja en País Vasco $(3,69)$. El hecho de que la razón más esgrimida sea por su simpatía y humor, nos lleva a considerar el valor del medio televisivo como entretenimiento frente a su poder formativo, hecho que ha de ser tenido en cuenta en la implementación de programas de alfabetización mediática e informacional.

Si nos referimos al tercer objetivo, como muestra la Tabla 3, la razón de identificación más alta se halla en intentan ver las cosas desde el punto de vista del personaje $(2,75)$ y la más baja en el argumento referido a porque le gustaría parecerse a él $(2,29)$. 
Tabla 3

Medias transculturales respecto a las razones por las que se identifican con el personaje que mejor les cae

\begin{tabular}{lcccc}
\hline \multicolumn{1}{c}{ REGIÓN } & $\begin{array}{c}\text { Intento ver las } \\
\text { cosas desde su } \\
\text { punto de vista }\end{array}$ & $\begin{array}{c}\text { Me preocupo } \\
\text { por lo que le } \\
\text { ocurre }\end{array}$ & $\begin{array}{c}\text { Yo mismo/a experimento } \\
\text { sus reacciones } \\
\text { emocionales }\end{array}$ & $\begin{array}{c}\text { Me gustaría } \\
\text { parecerme y actuar } \\
\text { como el/ella }\end{array}$ \\
\hline País Vasco & 2,67 & 2,37 & 2,30 & 2,08 \\
Aragón & 2,79 & 2,87 & 2,37 & 2,25 \\
Andalucía & 2,60 & 2,46 & 2,13 & 2,10 \\
Dublín & 2,85 & 2,83 & 2,52 & 2,69 \\
Jalisco & 2,52 & 2,15 & 2,01 & 1,93 \\
Cibao & 3,20 & 3,00 & 2,60 & 2,55 \\
Oruro & 2,80 & 2,66 & 2,59 & 2,55 \\
O’Higgins & 2,58 & 2,59 & 2,16 & 2,24 \\
\hline Total & 2,75 & 2,61 & 2,34 & 2,29
\end{tabular}

Ahora bien, una vez realizado el análisis de varianza para verificar las diferencias transculturales se puede comprobar que todas estas diferencias son, aunque moderadas, significativas (p'.001). No obstante, hay que exceptuar la razón referida a su preocupación por lo que le ocurre con $\left(\eta^{2}=.043\right)$ con la puntuación más alta en Cibao $(3,00)$ y la más baja en Jalisco $(2,15)$. Así mismo la razón que menor tamaño del efecto presenta, es la de ver las cosas desde su punto de vista $\left(\eta^{2}=.026\right)$ con la media más alta en Cibao $(3,20)$ y la más baja en Jalisco $(2,52)$. El hecho de que la media más alta se halle en «intento ver las cosas desde su punto de vista» explica cómo a través de la identificación con sus personajes preferidos pueden desarrollar su capacidad de empatía. Estos datos son de interés para una posible intervención educativa.

En cuanto al cuarto objetivo, como se puede observar en la Tabla 4, las puntuaciones más altas, tomados los datos en su conjunto, se encuentran en las consecuencias de las drogas y el alcohol $(2,94)$, seguido del ocio y la forma de divertirse de los jóvenes $(2,81)$ y la más baja en lo que ocurre en los centros escolares $(2,56)$. Comprobado si estas diferencias son estadísticamente significativas entre las regiones, todas ellas lo son (p'.001). Cabe destacar el tamaño del efecto por su cuantía en las siguientes respuestas: la televisión es realista en la manera de ser de chicos y chicas $\left(\eta^{2}=.085\right)$ con la puntuación más alta en Cibao $(\mathrm{M}=3,01)$ y las más baja en el País Vasco $(M=2,24)$, seguido de lo que ocurre en los centros escolares $\left(\eta^{2}=.065\right)$ con la puntuación más alta en O’Higgins $\mathrm{M}=(2,91)$ y la más baja en el País Vasco $(\mathrm{M}=2,23)$. Desde una perspectiva más cualitativa estos datos, a diferencia de los descritos en los objetivos anteriores, indican que la televisión, más allá del entretenimiento, debería ser considerada en sus aspectos formativos y, más en concreto, en el aspecto preventivo respecto al consumo de drogas y 
alcohol. En este sentido es importante señalar que la investigación indica que cuanto mayor es el realismo percibido mayor es el efecto incubado.

Tabla 4

Medias transculturales respecto al realismo percibido en la representación de temas de actualidad social

\begin{tabular}{|c|c|c|c|c|c|c|c|c|c|}
\hline \multirow[b]{2}{*}{ Realismo percibido } & \multicolumn{9}{|c|}{ REGIÓN } \\
\hline & $\begin{array}{l}\text { País } \\
\text { Vasco }\end{array}$ & Aragón & Andalucía & Dublín & Jalisco & Cibao & Oruro & O'Higgins & Total \\
\hline $\begin{array}{l}\text { La violencia en la } \\
\text { sociedad }\end{array}$ & 2,56 & 2,70 & 2,73 & 2,66 & 2,69 & 3,07 & 2,90 & 2,95 & 2,78 \\
\hline $\begin{array}{l}\text { El ocio y la forma } \\
\text { de divertirse }\end{array}$ & 2,52 & 2,73 & 2,88 & 2,54 & 2,89 & 3,03 & 2,96 & 2,92 & 2,81 \\
\hline $\begin{array}{l}\text { Las situaciones } \\
\text { familiares }\end{array}$ & 2,39 & 2,52 & 2,66 & 2,68 & 2,85 & 3,07 & 2,85 & 2,82 & 2,72 \\
\hline $\begin{array}{l}\text { Las relaciones } \\
\text { sexuales }\end{array}$ & 2,26 & 2,50 & 2,67 & 2,54 & 2,64 & 2,94 & 2,44 & 2,68 & 2,57 \\
\hline $\begin{array}{l}\text { Los patrones de } \\
\text { belleza. }\end{array}$ & 2,28 & 2,43 & 2,57 & 2,85 & 2,89 & 3,11 & 2,86 & 2,80 & 2,71 \\
\hline $\begin{array}{l}\text { Consecuencias } \\
\text { de las drogas y el } \\
\text { alcohol }\end{array}$ & 2,72 & 2,90 & 2,95 & 2,90 & 2,77 & 3,18 & 3,06 & 3,10 & 2,94 \\
\hline $\begin{array}{l}\text { La manera de ser } \\
\text { de los adolescentes }\end{array}$ & 2,24 & 2,40 & 2,63 & 2,74 & 2,73 & 3,01 & 2,94 & 2,85 & 2,68 \\
\hline $\begin{array}{l}\text { Situaciones } \\
\text { escolares }\end{array}$ & 2,23 & 2,34 & 2,66 & 2,32 & 2,60 & 2,79 & 2,71 & 2,91 & 2,56 \\
\hline
\end{tabular}

\section{CONCLUSIONES}

Analizados los datos en su globalidad y en referencia a los objetivos planteados, se puede afirmar que sí existen diferencias en los ocho contextos estudiados respecto a algunos indicadores del perfil de consumo televisivo. No obstante, estas diferencias al analizar los tamaños del efecto, no son tan grandes como cabría esperar. Si se realiza una lectura más cualitativa de los datos, este dato supone, al menos, poner en duda la consideración de que el medio televisivo es la causa de muchos de los males de la juventud, tal y como se ha hecho, en los estudios clásicos de televisión y socialización realizados en el marco de la teoría de los efectos y la teoría de la aculturación.

Los chicos y chicas que han participado en este trabajo, por otro lado, se dedican a otras actividades diferentes a ver la televisión. Cabe destacar que estar con la familia y estar con los amigos son las actividades a las que más horas dedican; es decir, a dos de los contextos más relevantes en el desarrollo del ciclo vital: la familia y los amigos. Apareciendo las horas 
de visionado de la televisión como una de las actividades a las que menos tiempo dedican. Sin embargo, es preocupante que la actividad de leer arroje unas de las medias más bajas junto con la utilización de videojuegos. No obstante, los datos aquí presentados, son bastante similares a los encontrados en los estudios realizados por el Ararteko (2009) y el INJUVE (2008), con la diferencia que ellos señalan escuchar música como la actividad a la que más tiempo se le dedica.

En cuanto a las razones esgrimidas para elegir a su personaje favorito, se puede constatar que una de las razones más señaladas es por su simpatía y humor y, a continuación, por su personalidad. Mientras que la razón de identificación que más estiman es la referida a intentar ver las cosas desde el punto de vista del personaje, y la más baja en el argumento referido a porque le gustaría parecerse a él; es decir, predomina la empatía cognitiva frente a la empatía conductual. Estos datos indican que tanto la empatía emocional referida a la capacidad de sentir lo mismo que los protagonistas, así como la empatía cognitiva referida a la capacidad de ponerse en el lugar del personaje y comprenderlo es posible activarla en el visionado de las narraciones televisivas. Esta conclusión merece ser tenida en cuenta desde el punto de vista educativo y nos da pistas para poder trabajar en el aula el desarrollo de la empatía a través de la identificación con los personajes en los distintos programas de alfabetización mediática (Cuervo y Medrano, 2013).

Cabe resaltar que la razón referida a su actitud no conformista y rebelde sea la opción con medias más bajas en el total de las regiones, seguida de por su atractivo físico. En principio y dada la etapa estudiada, cabría esperar que una actitud rebelde podría ser una buena razón de identificación. Así mismo, la razón referida al atractivo físico, en la que se ha hallado una media tan baja, hace reflexionar en la posibilidad de que el propio instrumento de recogida de datos haya favorecido la deseabilidad social puesto que estos datos no son congruentes con los hallados por Pérez y Torres (2012).

Respecto al realismo percibido, decir que son las consecuencias de las drogas y el alcohol, seguido del ocio y la forma de divertirse de los jóvenes las temáticas donde perciben un mayor realismo, mientras lo que ocurre en los centros escolares es la temática en la que menor realismo perciben. Estos datos ofrecen unas claves interesantes a la hora de poder diseñar intervenciones educativas que desarrollen estrategias de decodificación de los mensajes tanto explícitos como implícitos. Si tenemos en cuenta, de acuerdo a los datos de investigaciones previas, que cuanto mayor es el realismo percibido, mayor es el efecto incubado, deberíamos aprovechar las oportunidades que el medio ofrece para trabajar estas temáticas en los centros escolares a partir de sus preferencias televisivas. Es consecuencia, la propia televisión como agente socializador, se puede convertir en una herramienta 
para trabajar aquellas temáticas que los adolescentes perciben como realistas en la transmisión de sus mensajes y profundizar así en el desarrollo de las competencias emocionales del adolescente (Bisquerra y Pérez, 2007).

En este sentido, es preciso analizar los contenidos mediáticos y como indican Castillejo et al. (2011) y Pérez Alonso-Geta, (2010) advertir de los recursos que utilizan las empresas para incitar, por ejemplo, al consumo. Este hecho compromete a toda la comunidad educativa y no deberíamos atribuir a los medios de comunicación el empobrecimiento cultural de nuestra juventud, sino aprovechar su potencial como agente de socialización. Los datos aquí presentados no son alarmantes pero sí indican la posibilidad y conveniencia de trabajar las narraciones mediáticas en las aulas y desarrollar la capacidad formativa del medio más allá de su capacidad de entretenimiento.

El profesorado que de acuerdo a la investigación realizada por Jiménez (2007) en sus valoraciones diacrónicas otorga gran importancia a la formación debería ser preparado en el desarrollo e implantación de programas que favorezca la alfabetización mediática del alumnado. 


\section{REFERENCIAS BIBLIOGRÁFICAS}

Bisquerra, R. y Pérez, N. (2007). Las competencias emocionales. Educación $X X 1,10,61-82$.

Butcher, E. (2010). An audience reception analysis field study: Exploring second and later generation Latino viewers' perceived realism appraisals of Latino fictional television characters in English language television programs, Dissertation Abstracts International Section A, 70, 32-45.

Calvert, S. L. Murray, K. J. \& Conger, E. E. (2004). Heroic DVD portrayals: What US and Taiwanese adolescents admire and understand. Applied Developmental Psychology, 25, 699-716.

Cánovas, P y Sahuquillo, P. (2010). Educación familiar y mediación televisiva. Teoría de la educación, 22(1), 117-140.

Castillejo, J. L., Colom, A. J., Alonso, P. M., Rodriguez, T., Sarramona, J., Touriñán, J. M. y Vázquez, G. (2011). Educación para el consumo. Educación XX1, 14(1), 35-58.

Cohen, J. (2006). Audience identification with media characters. En J. Bryant y P. Vorderer (Eds.) Psychology of Entertainment (pp.183-197). Mahwah, NJ: Lawerence Erlbaum Associates.

Colom, A. J. (1998). El desarrollo sostenible y la educación para el consumo. Revista Pedagogía Social, 2, 31-49.

Cortina, A. (2004). La ética de los medios. Barcelona: Gedisa.

Cuervo, S. y Medrano, C. (2013). Alfabetizar en los medios de comunicación: más allá del desarrollo de competencias. Teoría de la Educación, 25(2), 111-131.
Elzo, J. (2006). Los jóvenes y la felicidad ¿dónde la buscan? ¿dónde la encuentran? Madrid: PPC.

Guarinos, V., Gordillo, I., Ramírez, M. M., Jiménez-Varea, J. y Hermida, A. (2010). másculinidades de ficción televisiva y retroalimentación online. Jóvenes y adolescentes en-red-ados fuera y dentro de Física o Química y El Internado. Recuperado de: http://www. aeic2010malaga.org/upload/ok/39.pdf

Hoffner, C. (1996). Children's wishful identification and parasocial interaction with favourite televisión characters. Journal of Broadcasting \& Electronic Media, 40(4), 25-37.

Informe extraordinario de la institución del Ararteko al Parlamento Vasco (2009). La transmisión de valores a menores Recuperado de: http://www. ararteko.net/RecursosWeb/DOCUMENTOS/1/1_1703_3.pdf

INJUVE (2008). Informe Juventud en España 2008. Observatorio de la Juventud. Madrid: Instituto Nacional de Juventud.

Martín, J. (1987). De los medios a las mediaciones. Barcelona: Gustavo Gili

Martín, M. (1998). Juventud y Consumo. Madrid: Instituto Nacional de Consumo.

Mattelart, A. y Mattelart, M. (1997). Historia de las teorías de la comunicación. Barcelona: Paidós.

Medrano, C. (2008). Televisión y Educación. Teoría de la educación, 20, 205-224.

Medrano, C., Aierbe, A. y Martínez de Morentin, J. I (2011). Valores percibidos en el medio televisivo por adolescentes en contextos transculturales. Comunicar, 37, 117-124. 
Medrano, C., Cortés, A., Aierbe, A. y Orejudo, S. (2010). Los programas y características de los personajes preferidos en el visionado de televisión. Cultura y Educación, 22, 3-20.

Medrano, C., Cortés, A. y Palacios, S. (2007). La televisión y el desarrollo de valores. Revista de Educación, 342, 307-328.

Negroponte, N. (1996). Ser Digital. México: Oceáno. Recuperado de: http:// www.Monografias.com/trabajos/influmcm/influmcm.shtml

Orozco, G., Navarro, E. y García Matilla, A. (2012). Desafíos educativos en tiempos de auto-comunicaciónmásiva. Comunicar, 38, 67-74.

Pérez, P. M. (2003). El impacto socializador de la TV en los niños y adolescentes de la Comunidad de Valencia. Valencia: Instituto de Creatividad e Innovaciones Educativas.

Pérez, P. M. (Dir.) (2010). Estudio sobre el comportamiento de los consumidores de juegos y juguetes (5-7años). Valores, perfiles y preferencias. Valencia: INICIE.

Pérez, R. y Torres, D. (2012). Representación de personajes, accesibilidad cognitiva y visionado televisivo en niños, niñas y adolescentes. Global Media Journal México, 9(17), 18-29.

Pindado, J. (2006). Los medios de comunicación y la construcción de la identidad adolescente. ZER, 21, 11-22.

Quick, B. L. (2009). The effects of viewing Grey's Anatomy on perceptions of doctors and patient satisfaction. Journal of Broadcasting \& Electronic Media, 53, 38-55.

Sartori, G. (1998). Homo videns. Madrid: Taurus.

Rodríguez, A., Medrano, C.; Aierbe, A. y Martínez de Morentin, J. I. (2013) Perfil de consumo televisivo y valores percibidos por los adolescentes: un estudio transcultural. Revista de Educación, 361, 513-538.

Sahuquillo, P. (2007). Algunas aportaciones teóricas a la influencia de la televisión en el proceso de socialización de la infancia. Teoría de la Educación, 19, 191-224.

Vera, J. (2001). Influencia educativa de los medios de comunicación social en la sociedad neoliberal. Teoría de la Educación, 13, 187-208.

\section{RECONOCIMIENTOS}

Investigación incluida en un proyecto I+D+I financiado por el Ministerio de Economía y Competitividad EDU 2012-36720, por el Gobierno Vasco como grupo consolidado IT688-13 y por la UFI de la UPV/EHU 11/04. 


\section{PERFIL ACADÉMICO Y PROFESIONAL DE LOS AUTORES}

Concepción Medrano, Catedrática del área de Psicología Evolutiva y de la Educación de la Universidad del País Vasco. Responsable de la Cátedra UNESCO de Comunicación y Valores Educativos en dicha Universidad y ha dirigido y codirigido distintos proyectos de investigación y tesis doctorales sobre valores y educación. Su actual línea de investigación se centra en los medios de comunicación y su relación con la formación de la identidad y los valores de la juventud.

Juan Ignacio Martínez de Morentin, Profesor Agregado del área de Psicología Evolutiva y de la Educación de la Universidad del País Vasco. Es Secretario Ejecutivo de la Cátedra UNESCO de Comunicación y Valores Educativos de dicha Universidad y ha desarrollado su labor docente e investigadora en temas relativos a la educación internacional, la psicomotricidad infantil, la construcción de la identidad, los valores educativos y las nuevas tecnologías en diseños curriculares on-line.

Pedro Apodaca, Catedrático del área de Métodos de Investigación en Educación de la Universidad del País Vasco. Su línea de investigación es la investigación evaluativa. Es asesor metodológico y ha sido responsable en dicha Universidad de los procesos de evaluación institucional. Igualmente ha colaborado con la Comisión Técnica del Consejo de Universidades en diversos trabajos. Es miembro permanente de la Cátedra UNESCO de Comunicación y Valores Educativos.

Dirección de los Autores: Universidad del País Vasco

Facultad de Filosofía y Ciencias de la

Educación

Avenida Tolosa, 70

20018 San Sebastián

Email: mariaconcepcion.medrano@ehu.es

juanignacio.demorentin@ehu.es

pedro.apodaca@ehu.es

Fecha Recepción del Artículo: 07. Febrero. 2012

Fecha Modificación Artículo: 26. Abril. 2012

Fecha Aceptación del Artículo: 06. Junio. 2012

Fecha Revisión para la publicación: 13. Junio. 2014 
